\title{
Automatic workflow for the classification of local DNA conformations
}

\author{
Petr Čech ${ }^{1,2}$, Jaromír Kukal ${ }^{2,3}$, Jiř́ Černý ${ }^{4}$, Bohdan Schneider ${ }^{4^{*}}$ and Daniel Svozil ${ }^{*}$
}

\begin{abstract}
Background: A growing number of crystal and NMR structures reveals a considerable structural polymorphism of DNA architecture going well beyond the usual image of a double helical molecule. DNA is highly variable with dinucleotide steps exhibiting a substantial flexibility in a sequence-dependent manner. An analysis of the conformational space of the DNA backbone and the enhancement of our understanding of the conformational dependencies in DNA are therefore important for full comprehension of DNA structural polymorphism.

Results: A detailed classification of local DNA conformations based on the technique of Fourier averaging was published in our previous work. However, this procedure requires a considerable amount of manual work. To overcome this limitation we developed an automatic classification method consisting of the combination of supervised and unsupervised approaches. A proposed workflow is composed of $k$-NN method followed by a nonhierarchical single-pass clustering algorithm. We applied this workflow to analyze $816 \mathrm{X}$-ray and 664 NMR DNA structures released till February 2013. We identified and annotated six new conformers, and we assigned four of these conformers to two structurally important DNA families: guanine quadruplexes and Holliday (four-way) junctions. We also compared populations of the assigned conformers in the dataset of $X$-ray and NMR structures.

Conclusions: In the present work we developed a machine learning workflow for the automatic classification of dinucleotide conformations. Dinucleotides with unassigned conformations can be either classified into one of already known 24 classes or they can be flagged as unclassifiable. The proposed machine learning workflow permits identification of new classes among so far unclassifiable data, and we identified and annotated six new conformations in the X-ray structures released since our previous analysis. The results illustrate the utility of machine learning approaches in the classification of local DNA conformations.
\end{abstract}

Keywords: DNA, Dinucleotide conformation, Classification, Machine learning, Neural network, RBF, MLP, k-NN, Regularized regression, Cluster analysis

\section{Background}

The antiparallel double helical structure of DNA and its self-recognition form the basis for the conservation and the transfer of genetic information. The model of the "canonical"B-DNA form proposed by Watson and Crick [1] has later been enriched by detailed structural data from single-crystal structures of the biologically prevailing B-form [2] and of its kin right-handed A-form $[3,4]$. In addition, the first DNA single crystal [5] revealed atomic details of a third major form of a DNA

\footnotetext{
*Correspondence: bohdan@img.cas.cz; svozild@vscht.cz

${ }^{4}$ Institute of Biotechnology AS CR, v. v. i., Vídeňská 1083, Prague 414200 ,

Czech republic

${ }^{1}$ Laboratory of Informatics and Chemistry, ICT Prague, Technická 5, Prague 6 166 28, Czech republic

Full list of author information is available at the end of the article
}

double helix, left-handed Z-DNA. The atomic resolution structures of B-DNA duplexes [6] revealed the existence of sequence-dependent structural deviations which provide the required specificity for DNA recognition by proteins and drugs [7]. The association of DNA with proteins is known to induce a local deformation of the B-form toward the A-form [8-13] in various protein-DNA complexes such as, e.g. high mobility group (HMG) proteins [14], trp repressor/operator complex [15], TATA box binding protein [16-18], HIV-1 reverse transcriptase [19], various DNA polymerases [20-23], zinc finger protein [24], hyperthermophile Sac7d protein [25], and EcoRV endonuclease [26-28]. Along the transition pathway between the B- and A-forms [29] various intermediate B-to-A conformations were identified [9,30-32]. The importance of conformational sub- 
states of the DNA backbone for protein binding to the minor groove was suggested by several analyses [13,33,34]. Besides the A-, B- and Z-forms, DNA can also adopt other biologically relevant structures, such as single-stranded hairpins [35], triple helices [36], three- and four-way junctions [37,38], four-stranded G-quadruplexes [39] or parallel helices [40]. Their existence indicates that DNA structure is much more polymorphic than it might be deduced from the misleading simplicity of the canonical B-DNA duplex.

The base morphology in a DNA double helix is commonly described [12,41-46] by parameters giving mutual position between bases in a base-pair (e.g., propeller twist or stagger) and in a base-step (e.g. rise or twist) [47]. The same parameters can also be used for other unusual DNA structures such as triple helices [48-50], G-quadruplexes [51] or three- and four-way junctions $[52,53]$. In addition, for the last two groups of structures additional specific parameters such as the G-quartet planarity [54] or the angle between the junction arms [55] were also defined. Another set of quantitative measures that can be used to characterize secondary structure of DNA are backbone torsional angles $\alpha, \beta, \gamma, \delta, \varepsilon, \zeta$ together with the glycosidic torsion $\chi$ [56]. Though the relationship between the phosphodiester backbone states and local distortions of DNA double helix was described in the ' 80 and ' 90 s $[57,58]$, the backbone was regarded as a passive link holding bases at their positions in several early analyses $[7,59,60]$. However, nowadays it is clear that the backbone must be considered as an active dynamic element while defining the conformational properties of double-helical DNA [34,61-69]. The main role of the backbone is in restricting the conformational space available for the placement of bases, and in steric coupling of the adjacent base steps [61]. An overall conformational flexibility of DNA thus results from the interplay between the optimal base positions and the preferred conformations of the sugar-phosphate backbone. An increasing number and quality of DNA structures led to several detailed analyses of the conformational space of the DNA backbone, most of these studies have been based on crystal structures [32,70-73] but structures determined by various solution-based techniques of NMR spectroscopy have also contributed significantly to our understanding of biology of nucleic acids [74-76]. NMR methods were successfully applied to study a dynamics of DNA phosphodiester backbone in solution [77-82], NMR studies also provide evidence for the BII states in solution and help to unravel a role of the phosphorus atom in a BIBII transition [68,83-87].

To uncover a potential role of the sugar-phosphate backbone in the DNA structural polymorphism we have analyzed a set of carefully selected double-helical structures of naked and protein bound DNA resolved at high resolution $(\leq 1.9 \AA)$ [32]. We have identified all the known major conformers (AI, AII, BI, BII, and ZI and ZII) as well as several minor conformations corresponding to various transitional states between the $\mathrm{B}$ and $\mathrm{A}$ forms. The investigation was based on the technique of Fourier averaging in combination with a cluster analysis applied previously on the annotation of RNA conformers [88]. The main disadvantage of the Fourier averaging approach is that it requires a considerable amount of manual work [32]. To automate this process we introduce here a machine learning workflow that deals with two following tasks:

1. Classify data points into one of the existing classes.

2. Recognize data points that cannot be classified and identify new possible conformational classes.

The first task is accomplished by the application of the supervised machine learning approaches. In supervised algorithms a classification function is inferred from the labeled training data (i.e. each data point must be assigned to an appropriate class). As a training set we used previously published classification of DNA local conformers [32]. In the present study we applied and compared several supervised methods: multi-layer perceptron (MLP) neural network, radial basis function (RBF) neural network, $k$ nearest neighbors $(k-\mathrm{NN})$, and ridge regression (RR). The best method ( $k-\mathrm{NN})$ not only achieves high classification accuracy, but also allows identifying conformers that cannot be assigned to any of the known classes. Such conformers were subsequently investigated for the presence of new clusters using a modified clustering method based on a leader algorithm [89]. The proposed classification workflow (Figure 1) was applied on the analysis of X-ray data updated by structures released after 18 July 2005, and of NMR data released until 15 February 2013.

\section{Methods}

\section{Data sets}

For the development of the machine learning workflow we used a previousy published data set [32] consisting of 7,739 dinucleotides collected from 389 high quality crystal structures with a resolution of $1.9 \AA$ or better and from 58 structures with unusual topologies (G-quadruplexes, i-motif, three- and four-way junctions, etc.). These structures were released into the Nucleic Acid Database [90] before 19 July 2005. In this data set we originally identified 119 conformational families. To reduce their number for the classification purposes, we critically evaluated the data for the presence of outliers and for the size and quality of the clusters. 419 outliers were removed, and the number of conformationally distinct families was condensed into 18 classes (Table 1) resulting in a data set consisting of 7,320 data points. 


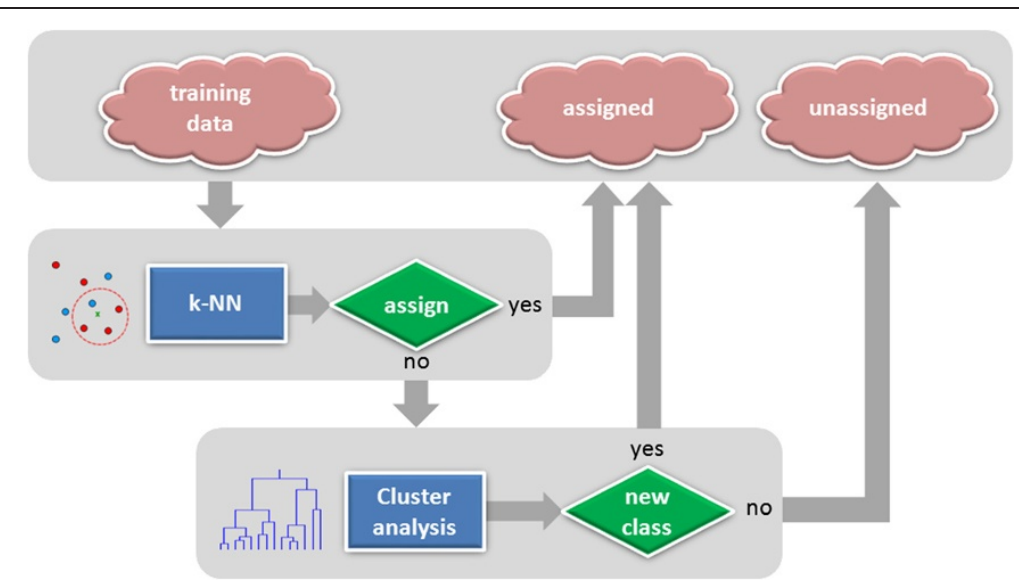

Figure $1 \mathrm{~A}$ workflow of the classification of local DNA conformations. $k$-NN uses 11 neighbors (parameter $k$ ). A threshold $v_{\text {crit }}=0.001$ (see explanation in the Methods section of the manuscript) was used to distinguish between data points that can be assigned to some of existing classes or cannot be assigned at all. Cluster analysis uses a modified version of the single-pass nonhierarchical leader algorithm [89].

These data were split into 4,567 dinucleotide units (DatasetF) classified previously by the Fourier clustering, and into 2,753 dinucleotides that were not assigned to any class in our previous work [32]. A stratified sampling was used to divide the DatasetF into the training (DatasetF_train, see Additional file 1) and test (DatasetF_test, see Additional file 1) sets in the ratio 80:20. DatasetF_train was used for classifier's learning, and the DatasetF_test was used for assessing its performance. Training set contains 3,651 data points, and test set contains 906 data points. In a stratified division each of the classes is sampled with the ratio present in the total population. For example, class number 54 (BI-DNA, see Table 1) covers $42.5 \%$ of the total population, and is present in this proportion also in DatasetF_train and in DatasetF_test.

Our machine learning classification workflow was then applied to $427 \mathrm{X}$-ray structures, resolved with a crystallographic resolution of $1.9 \AA$ or better, and released between 18 July 2005 and 15 February 2013, which contained 8,708 dinucleotides, and to 664 NMR structures released before 15 February 2013, which contained 12,300 dinucleotides.

Table 1 Characteristics of the local B-DNA backbone conformations used in the present work

\begin{tabular}{|c|c|c|c|c|c|c|c|c|c|c|c|}
\hline Class ID & Description & $\mathbf{N}$ & $\delta$ & $\varepsilon$ & $\zeta$ & $a+1$ & $\beta+1$ & $\gamma+1$ & $\delta+1$ & $x$ & $x+1$ \\
\hline 8 & A-DNA & 325 & 83 & 205 & 287 & 294 & 174 & 54 & 83 & 199 & 202 \\
\hline 13 & A-DNA, Bl-like $x, x+1$ & 196 & 89 & 201 & 275 & 294 & 162 & 54 & 89 & 244 & 244 \\
\hline 19 & A-DNA, $a+1 / \gamma+1$ crank $(\mathrm{t} / \mathrm{t})$ & 65 & 82 & 195 & 291 & 149 & 194 & 182 & 87 & 204 & 188 \\
\hline 32 & Bl-to-A, O4'-endo $\delta+1$ & 266 & 129 & 186 & 264 & 295 & 170 & 52 & 99 & 247 & 233 \\
\hline 41 & A-to-B, $>C 3^{\prime}$-endo $\delta, C 2$-endo $\delta+1$ & 215 & 90 & 196 & 280 & 299 & 179 & 55 & 142 & 222 & 256 \\
\hline 50 & $\mathrm{Bl}, \mathrm{C} 1$ '-exo $\delta+1$ & 392 & 129 & 181 & 265 & 300 & 177 & 50 & 123 & 246 & 245 \\
\hline 54 & $\mathrm{Bl}$ & 1942 & 136 & 183 & 259 & 303 & 181 & 44 & 138 & 252 & 259 \\
\hline 86 & Bll variation in complexes & 314 & 140 & 201 & 216 & 314 & 153 & 46 & 140 & 262 & 253 \\
\hline 96 & Bll & 539 & 143 & 245 & 170 & 297 & 141 & 46 & 141 & 271 & 257 \\
\hline 109 & Bll-to-A, C3'-endo $\delta+1$ & 20 & 142 & 213 & 181 & 297 & 139 & 52 & 90 & 273 & 207 \\
\hline 110 & Bll-to-A, $a+1 / \gamma+1$ crank $(g+/ t)$, high $\beta+1$ & 9 & 146 & 257 & 186 & 60 & 224 & 196 & 90 & 260 & 200 \\
\hline 116 & $\mathrm{Bl}, a+1 / \gamma+1$ crank (g+/g-) & 158 & 140 & 194 & 247 & 31 & 197 & 294 & 150 & 253 & 253 \\
\hline 119 & Bl mismatches, syn/anti & 11 & 144 & 189 & 266 & 303 & 167 & 53 & 138 & 70 & 259 \\
\hline 121 & A-to-B, $>C 3^{\prime}$-endo $\delta$, anti/syn & 19 & 100 & 209 & 278 & 295 & 174 & 54 & 128 & 243 & 67 \\
\hline 122 & Bl mismatches, anti/syn, $a+1 / \gamma+1$ crank ( $\mathrm{g}+/ \mathrm{g}-)$ & 8 & 137 & 196 & 225 & 33 & 187 & 295 & 145 & 257 & 70 \\
\hline 123 & Z-DNA, Y-R & 21 & 147 & 264 & 76 & 66 & 186 & 179 & 95 & 205 & 61 \\
\hline 124 & Z-DNA, R-Y ZI & 49 & 96 & 242 & 295 & 209 & 231 & 55 & 144 & 63 & 205 \\
\hline 126 & Z-DNA, R-Y ZII & 18 & 95 & 187 & 63 & 169 & 162 & 44 & 144 & 58 & 213 \\
\hline
\end{tabular}

"Class ID" is the symbolic label of the class. "Description "is a short annotation of the class. " $\mathrm{N}$ " is the number of suites (dinucleotides) with the given class membership. Values of torsions represent the arithmetic means for individual classes. Torsions are defined in Figure 2. 
For our analysis a concept of a "suite" [91] was adopted. "Suite" is a conformational subset of a dinucleotide unit (Figure 2) going from sugar to sugar and consisting of 7 backbone torsions $(\delta, \varepsilon, \zeta, \alpha+1, \beta+1, \gamma+1, \delta+1)$. The analysis also includes two glycosidic angles $X$ and $X+1$. Each data point is therefore represented by a vector composed of 9 torsion angles. In the following text we also use the convention [56] by which it is common to describe the backbone torsional angles of $\sim 60^{\circ}$ as gauche+ $(\mathrm{g}+)$, of $\sim 300^{\circ}$ as gauche- $(g-)$, and of $\sim 180^{\circ}$ as trans $(t)$. For glycosidic torsion $X$ following regions are commonly used: $\operatorname{syn}\left(0^{\circ}-90^{\circ}\right)$, anti $\left(240^{\circ}-180^{\circ}\right)$, and low anti $\left(\sim 200^{\circ}\right)$.

\section{Data preprocessing}

The input data (raw angle values from the $0^{\circ}-360^{\circ}$ interval) were used either directly (in $k$-NN method) or they were normalized using one of the following methods:

1. In a geometric preprocessing each torsion was transformed from the space of dihedral angle $\theta \in\{\delta$, $\varepsilon, \zeta, \alpha+1, \beta+1, \gamma+1, \delta+1, \chi, \chi+1\}$ to the linear metric coordinate space built up by the series of trigonometric functions $\{\sin n \theta, \cos n \theta\}$ with the geometric order parameter $n=1, \ldots, D$. This preprocessing method accounts for the circular character of angular data [92,93], however it increases the length of the input vector from 9 to

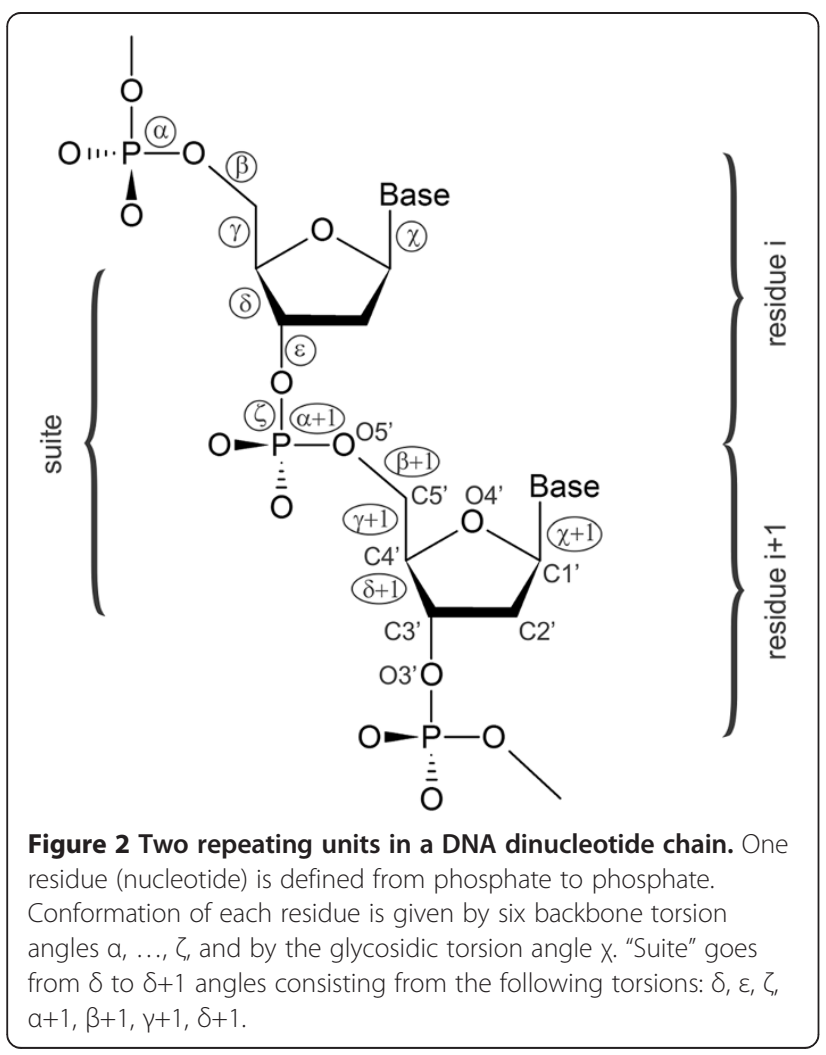

$2 D \times 9$. This preprocessing was used in RR, MLP and RBF methods.

2. In a linear preprocessing each angle was converted into the $\langle-1,1\rangle$ range. This conversion increases the performance in the Matlab environment that was used for all neural networks simulations. This preprocessing was used in MLP and RBF methods.

Depending on the classification method, the output data (i.e., the class membership of individual data points) were encoded in two different ways:

1. The original class numbering (see Table 1) was used in $k$-NN.

2. Classes were renumbered to the interval 1-18, and the class membership was then encoded as a binary vector of the length 18 . This encoding was used in RR, MLP and RBF methods.

\section{Training and cross-validation}

Each classifier is characterized by one or more parameters that are tuned to capture the underlying relationships in the training data set, and that influence the ability of an algorithm to perform accurately on new, previously unseen examples (the generalization ability). The combination of one particular method (e.g. MLP neural network) with particular values of parameters (e.g. number of hidden neurons equaling to 10) is designated as a model. The most appropriate values of the parameters were chosen using a well established method of $k$-fold cross-validation. In $k$-fold crossvalidation, a training set is divided into $k$ parts. A classifier is trained $k$-times, each time leaving out one of the subsets (the so-called validation set), which is used to assess the classifier's performance. At the end, the final validation error is obtained as the average of all errors from $k$ individual validation runs. In the present work a 10 -fold cross-validation was adopted using the stratified division of the DatasetF_train. The quality of the trained model was evaluated by the Mean Squared Error of Validation $M S E_{\text {validation. }}$

$$
M S E_{\text {validation }}={ }_{n}^{1} \sum_{i=1}^{n}\left(P_{i}-T_{i}\right)^{2}
$$

where $P_{i}$ is the predicted class membership and $T_{i}$ is the known class membership. To smooth out possible biases caused by an unfavourable random data set division, the 10 -fold cross-validation was repeated 10 times, and the final $M S E_{\text {validation }}$ was obtained as an average of validation errors from all individual runs. A model with the lowest $M S E_{\text {validation }}$ represents the "best" model. Once it was identified the final model was trained using the whole DatasetF_train. The 
quality of individual classifiers was compared using the $M S E_{\text {test }}$ calculated for the DatasetF_test.

\section{Classifiers}

\section{A multi-layer perceptron (MLP)}

MLP represents the most common architecture of neural networks. It consists of simple processing units (neurons) arranged into three or more layers: one input layer, one or more hidden layers, and one output layer. Every neuron in one layer is connected to every neuron in the following layer, and no intra-layer connections exist. The strength of neuron connections is represented by numerical weight values. The weights are free variables of the system which are determined during the training phase. Neurons transform a numerical input to an output value via the transfer function. In the present work, a two-layer perceptron consisting of one input, one hidden and one output layer was used. Several transfer functions were tested: linear, log-sigmoid and tan-sigmoid. Log-sigmoid function is given as

$$
\log \operatorname{sig}(u)=\frac{1}{1+e^{-u}}
$$

and tan-sigmoid function is given as

$$
\operatorname{tansig}(\boldsymbol{u})=\frac{\boldsymbol{e}^{2 u}-1}{\boldsymbol{e}^{2 u}+1}
$$

where a potential $\mu$ of a neuron is given as $u=\sum_{i} w_{i} x_{i}-9$, $\bar{x}=\left[x_{1}, \ldots, x_{i}\right]$ is the input vector, $\bar{w}=\left[w_{1}, \ldots, w_{i}\right]$ is the weight vector, and $\vartheta$ is the neuron's bias (threshold). As the neuron's input goes from negative to positive infinity, the log-sigmoid function generates outputs between 0 and 1 , and the tan-sigmoid function generates outputs between -1 and 1 .

\section{Radial basis function network (RBF)}

$\mathrm{RBF}$ is also a two-layer neural network. The input layer serves only as a mediator in passing a signal to the hidden layer. While MLP is based on units which compute a non-linear function of the scalar product of the input vector and a weight vector, in RBF the activation of a hidden unit is determined by the distance between the input vector and a prototype vector. Each hidden neuron modulates the input signal by the Gaussian transfer function called radial basis function (RBF). Each RBF is characterized by two parameters: by its center (position) representing the prototype vector, and by its radius (spread). The centers and spreads are determined by the training process. When presented with the input vector $\bar{x}$, the Euclidean distance of the input from the neuron's center is computed by the hidden neuron, and the RBF kernel function is applied to this distance. The output from the network is constructed as a weighted sum of the RBF's outputs. The weights are also determined in the training phase. While MLP separate the classes by using hidden neurons which form hyperplanes in the input space yielding a global approximation, RBF networks model the separate class distributions by local radial basis functions.

\section{k-nearest neighbor ( $k$-NN)}

In $k$-NN method objects are classified based on the class of their nearest neighbors. A new point is assigned to the majority class among the $k$ nearest points. $k-\mathrm{NN}$ is a lazy algorithm meaning that there is no explicit training phase, it makes no generalization (i.e. no underlying model of the class membership is constructed), and the decision is based on the entire training data set which must be available during the prediction phase. Euclidean distance is used as a measure of the proximity of two data points. To get the Euclidean distance between two torsion angle vectors the similarity vector $\bar{s}$ must be calculated first. Its elements $s_{i}$ are distances between individual components of compared vectors. To correctly calculate the similarity vector $\overline{\mathrm{s}}$ the circularity of the angular data must be taken into account. The distance $s_{i}$ between two angles $\phi$ and $\psi$ is given as [94]

$$
\mathbf{s}_{\mathbf{i}}=180-|180-| \phi-\boldsymbol{\psi}||
$$

where both $\phi$ and $\psi$ angles are given in degrees. The $\mathrm{Eu}-$ clidean distance $\mathrm{d}$ is calculated as

$$
\mathbf{d}=\sqrt{\sum_{\mathbf{i}} \mathbf{s}_{\mathbf{i}}^{2}}
$$

In $k$-NN approach, the number of nearest neighbors $k$ represents the only adjustable parameter of the method. The class membership of $k$ nearest neighbors was used to assign the class of the classified point. To take into account a fact that near neighbors influence the resulting class membership more than the distant ones contributions of the neighbors were weighted by $1 / \mathrm{d}^{2}$. The point was assigned to the class with the highest sum of weighted contributions. However, if this sum was less than a threshold $\mathrm{v}_{\text {crit }}=0.001$, the data point was declared as unclassified. The value of $\mathrm{v}_{\text {crit }}$ was obtained empirically and, based on our experience, optimally balances the accuracy of the method and the number of unassigned points in the dataset.

\section{Regularized regression (RR)}

RR [95] is a standard statistical method of linear modeling and parameter identification. In RR pattern set is represented as a pair $(X, Y)$, where $X$ is an input matrix of the size $m \times n, Y^{*}$ is an output matrix of a size $m \times N$, $m$ is the number patterns, $n$ is the number of inputs and $N$ is the number of outputs. Ridge regression penalizes the size of the regression coefficients by the penalty 
calculated as a weight matrix $W=\left(X^{T} X+\lambda I\right)^{-1} X^{T} Y^{*}$ where $\lambda \geq 0$ is a regularization parameter and $I$ is an $n \times$ $n$ identity matrix. If the matrix $Y^{\prime \prime}$ represents the class membership, the RR response is calculated as $Y=X W$ and the $i$ th pattern is assigned to the $j$ th class for which the $y_{i, j}$ element is maximal. Main advantages of RR are fast learning procedure and ability to solve ill-posed problems with a high number of possibly dependent explanatory variables. The disadvantage of RR is the linearity of the underlying model. However, the linearity limitation can be suppressed by an appropriate nonlinear preprocessing of the data.

\section{Comparing classifiers}

The quality of classification models is assessed by various measures based on the counts of correctly and incorrectly predicted test data [96]. Such information can be tabulated as a confusion matrix. Each row of the matrix represents the instances in the actual class, and each column represents the instances in the predicted class. To compare the performance of various classification models this matrix is usually boiled down to the single number. In the present work two such performance metrics - accuracy and $\mathrm{k}$ coefficient - were utilized. Accuracy is defined as a percentage of correctly classified data points, i.e. the main diagonal in the confusion matrix is summed (this gives the number of correctly classified data points - true positives TP) and the sum is divided by the total number of observations $\mathrm{N}$ :

$$
\text { accuracy }=\frac{\mathbf{T P}}{\mathbf{N}} \cdot 100
$$

The disadvantage of the accuracy is that it does not reveal if an error is evenly distributed between classes or if some classes are really bad and some really good. To include this information the $\kappa$ coefficient [97] takes into account also the off-diagonal elements

$$
\mathbf{K}=\frac{\mathbf{N} \times \sum_{\mathbf{i}=1}^{\mathbf{n}} \mathbf{x}_{\mathbf{i i}}-\sum_{\mathbf{i}=1}^{\mathbf{n}}\left(\mathbf{x}_{\mathbf{i}+} \times \mathbf{x}_{+\mathbf{i}}\right)}{\mathbf{N}^{2}-\sum_{\mathbf{i}=1}^{\mathbf{N}}\left(\mathbf{x}_{\mathbf{i}+} \times \mathbf{x}_{+\mathbf{i}}\right)}
$$

where $n$ is the number of rows in the confusion matrix, $x_{i i}$ is the number of observations in row $i$ and column $i$, $x_{i+}$ and $x_{+i}$ are the marginal totals of row $i$ and column $i$, respectively, and $N$ is the total number of observations. $\kappa$ coefficient measures the improvement of classifier's predictions over a purely random assignment to classes.

\section{Cluster analysis}

The main objective of clustering is to find a grouping of similar objects within a data [98]. The objects are not labeled, and cluster analysis belongs between unsupervised methods. In the present work we used a nonhierarchical single-pass method that works on the basis of a single scan of the data set. The most common single-pass algorithm is called the leader algorithm [89] which is simple to implement and very fast. However, its major drawback is that it is order dependent meaning that if the compounds are rearranged in a different order then the resulting clusters can be different [89]. Therefore we developed a modified leader algorithm which retains high speed, and is order independent. The used algorithm consists of the following steps to provide a set of clusters:

1. Set the number of existing clusters to zero.

2. For each data point (i.e., set of nine torsions characterizing a given dinucleotide) $D_{i}$

- Start new cluster $C_{i}$

- Calculate a neighborhood of $D_{i}$

- Go through all data points except $D_{i}$. Data points belonging to the neighborhood of $D_{i}$ are appended to the cluster $C_{i}$

3. Remove duplicated clusters getting a set of unique clusters (a unique set).

4. Repeat until the unique set is empty

- Identify the biggest cluster $B_{i}$ in the unique set

- If the size of $B_{i}$ is higher than predefined threshold append $B_{i}$ to the final set of clusters

- Identify all clusters that overlap with $B_{i}$

- Remove $B_{i}$ and all overlapping clusters from the unique set

In point 2. a dinucleotide belongs to the neighborhood of $D_{i}$ if its torsion deviates from $D_{i}$ by no more than $20^{\circ}$ for $\alpha, \varepsilon, \zeta$, and $\chi, 30^{\circ}$ for $\beta, 15^{\circ}$ for $\gamma$, and $10^{\circ}$ for $\delta$. These intervals were selected on the empirical basis reflecting common conformational variability ("stiffness") of the individual torsion angles. A cluster is defined by at least six points in the presented study, which gives a value of a threshold in point 4 .

\section{Results and discussion}

\section{Optimal parameters of the classification methods}

In MLP, we determined the input preprocessing method, the number of hidden neurons and the type of transfer function by the 10 -fold cross-validation. The number of hidden neurons varied between 10 and 60 with the step of 2 . We performed the cross-validation with every possible combination of linear, log-sigmoid and tan-sigmoid transfer functions using either linear or geometric preprocessing. The order parameter $n$ of the geometric preprocessing was cross-validated, its values varied from 1 to 10 by one. The optimal MLP model uses the geometric preprocessing with $n=1$ (i.e. the input vector 
consists of $2 \times=18$ components), has 22 neurons in a hidden layer, and uses log-sigmoid (Equation 2) transfer function at hidden neurons and tan-sigmoid (Equation 3) transfer function at output neurons.

In $\mathrm{RBF}$, the input preprocessing method, the number of hidden neurons and the optimum spread of the Gaussians on hidden neurons were recognized using the 10 -fold cross-validation. The order parameter $n$ of the geometric preprocessing varied from 1 to 10 by one, the spread varied in the interval of 0.05 and 0.025 with the step of 0.01 , and the number of hidden neurons varied by one between 10 and 50 . The optimal RBF utilizes a geometric preprocessing with $\mathrm{n}=1$ and has 18 hidden neurons with the spread of 0.15 .

In $k$-NN, the number of nearest neighbours $k$ was varied between 1 and 50. Its optimum value found by 10fold cross-validation is equal to 11 .

In $R R, 10$-fold cross-validation was used to set the order $k$ of the geometric preprocessing and the regularization parameter $\lambda$. The order $k$ was varied between 1 and 10 by one, and the regularization parameter $\lambda$ was set either to 0 or it was altered by factors of 10 from $10^{-6}$ to $10^{-3}$. The optimum order of the geometric preprocessing is 6 which leads to the increase of the length of the input vector from 9 to $2 \times 6 \times 9=108$. The optimum regularization parameter $\lambda$ is zero. With this regularization parameter the ridge regression is equivalent to the standard linear regression.

\section{Performance of the classification methods}

The accuracy of individual classification methods is summarized in the Table 2 and the confusion matrices showing the class predictions given by individual classifiers are available in the Additional file 2.

The best performing classifier both in terms of accuracy and $\kappa$ coefficient is the multi-layer perceptron MLP followed by the $k$-nearest neighbors $k$-NN and by the ridge regression RR. MLP and $k$-NN are both non-linear

\begin{tabular}{|c|c|c|c|c|}
\hline & MLP & RBF & $k-\mathrm{NN}$ & RR \\
\hline accuracy [\%] & 97,35 & 88,41 & 96,58 & 94,92 \\
\hline K coefficient & 0,966 & 0,845 & 0,956 & 0,934 \\
\hline
\end{tabular}

These were evaluated using test set (DatasetF_test). The MLP model uses geometric preprocessing with the order $k=1$, has 22 hidden neurons with the log-sigmoid transfer function and output neurons use the tan-sigmoid transfer function. The best RBF model uses geometric preprocessing with the order $k=1$, has 18 hidden neurons with the spread of 0.15 . The optimal value of $k$ in $k$-NN is 11 . In RR, the optimal regularization parameter $\lambda$ is zero, and the order of the geometric preprocessing expansion $k$ is 6 . classifiers, while RR represents a linear method. The penalization of the coefficients in the ridge regression is not necessary (regularization parameter $\lambda$ is zero), and the ridge regression is therefore reduced to the standard linear regression. However, RR performs similarly to nonlinear methods due to the sophisticated preprocessing method motivated by the geometrical nature of the input angular data. A careful inspection of the confusion matrices (Additional file 2) reveals that the decrease in accuracy is caused mainly by misassignment between two pairs of classes: points belonging to the class 50 (BI conformers with the second sugar at the $\mathrm{C}^{\text {'}}$-exo conformation, see Table 1) can be assigned to the class 54 (BI conformers, see Table 1), and points belonging to the class 32 (BI-to-A conformers with the second sugar at the O4'-endo conformation, see Table 1) can be assigned to the class 50. Classes 54 and 50 are distinguished mainly by a slight difference in the sugar pucker at both deoxyriboses $\left(7^{\circ}\right.$ in $\delta$ and $15^{\circ}$ in $\delta+1$, see Table 1 ), the conformational transition between these classes is continuous and a limited blending of the conformers can be expected. Similar behavior show also classes 50 and 32 as they differ primarily in the $\delta+1$ torsion, the difference is $24^{\circ}$ (see Table 1 ).

A poor performance of RBF comes as a surprise. Reason for this behavior can be that the classification boundary in RBF is constructed in a local manner, while MLP and RR are global methods and in $k$-NN the classification boundary is not constructed explicitly. However, an RBF confusion matrix (Additional file 2) reveals that the decrease in accuracy is also caused by misassignments between classes 50 and 51 (51 misassigned points) and between classes 32 and 50 (15 misassigned points). As explained above, certain extent of the mixing of these conformers can be expected, and we can thus conclude that a lower accuracy of the RBF network is only seeming and RBF performs similarly as the other investigated methods.

Of the studied methods, $k$-NN offers one important advantage: it allows to discriminate between conformations that can be assigned to one of the pre-defined classes and between the conformations for which such a class does not exist. From this reason we propose $k$-NN as a method of choice for the classification of local conformations in nucleic acids.

\section{Analysis of the newly characterized conformers $X$-ray structures}

We analyzed 2,753 dinucleotides unassigned to any class in our previous work [32], and 8,708 dinucleotides from 427 X-ray structures released between 18 July 2005 and 15 February 2013. Utilizing the $k$-NN approach (with $k=11$ and $\left.\mathrm{v}_{\text {crit }}=0.001\right)$ we assigned 10,510 (91\%) dinucleotides to one of 18 possible (Table 1) classes. Applying a clustering procedure on remaining 951 unassigned dinucleotides 
representing results of incorrect refinement of the crystallographic model or yet unidentified clusters we identified 6 new conformational classes (Table 3). A data set containing all X-ray structures analyzed in the present work can be found in Additional file 1.

Four of six new conformers can be found exclusively in two functionally distinct types of non-double helical structures. Conformer 115 occurs in four-way (Holliday) junctions, and conformers 97,113 , and 114 are found in guanine quadruplexes of the Oxytricha nova telomere. Other two conformers (117 and 35) are found in various DNA-protein complexes. A detailed description of new conformations is given in the following paragraphs.

\section{Conformations 97, 113 and 114}

These conformations are found exclusively in guanine quadruplexes (G-quadruplexes) of the O. nova telomere. G-quadruplexes represent biologically very interesting non-canonical DNA structures [39,99]. G-rich sequences, in which G-quadruplexes often appear, are abundant in the genome, and are found e.g. in telomeric regions [100], immonugloboluline switch regions [101] or gene promoter regions [102]. G-quadruplex of $O$. nova telomere is a well-studied $[103,104]$ example of bimolecular, antiparallel quadruplex with the sequence $d$ $\left(G_{4} T_{4} G_{4}\right)_{2}$. A core structural element of G-quadruplexes are planar G-quartets (also termed a G-tetrads) that stack on top of each other. They are connected by loops of variable length and composition whose variations lead to a wide variety of topologies of G-quadruplexes.

In our previous work [32] we were able to match several dinucleotides in $O$. nova G-quadruplex with distinct types of conformers and new conformers 97, 113, and 114 identified in the present work further enhance this structural annotation (Figure 3(a), (b) and (c)). Class 113 is a highly distorted BI-like conformation with $\varepsilon / \zeta$ in $t / g+$, $\alpha+1 / \gamma+1$ switched into $g+/ t$ values and $\chi+1$ in the syn region $\left(\sim 68^{\circ}\right)$. Conformer 114 represents a BI-like conformation with anti/syn arrangement of $X$ and $X+1$ torsions, high $\beta\left(\sim 260^{\circ}\right)$, and unusual $g-\left(\sim 300^{\circ}\right)$ value for $\gamma+1$ torsion. Conformation 97 represents a BII conformation with $\alpha+1 / \gamma+1$ switched into $t / g+$ values, and with $\chi+1$ in low anti region $\left(\sim 185^{\circ}\right)$.

By analyzing crystal (1L1H [107], 1PH4, 1PH6, 1PH8 [108], 1JB7 [103], 2HBN [109], 3EUM [106], 3NYP [110]) and NMR (156D [111], 230D [112], 1K4X [113], 2AKG [114]) structures we were able to construct a consensus conformational map (Figure 3(d) and (e)) showing the succession of conformers in the O. nova Gquadruplex. From the studied pool of structures, four (3EUM, 3NYP, 1L1H, and 3NZ7) represent a complex of the G-quadruplex with a drug acridine, while the rest are not complexed. Acridine binds to the quadruplex within its $\mathrm{T}_{4}$ loop in chain A [107] influencing a conformation of the whole $\mathrm{T}_{4}$ loop. Thus, we have considered naked and acridine-complexed structures separately in our analysis.

The consensus conformational map of the naked Gquadruplex is shown in Figure 3(d), and that of the complex with the acridine in Figure 3(e). Common to both are conformations present in the chain B, and in the Gtracts of the chain A. O. nova G-tracts exhibit a wellknown 5'-syn-anti-syn-3' pattern [107] of guanine glycosidic torsion angles manifested by alternating conformations 119-122-119 in the G1G2G3G4 sequence, and conformations 119-114-119 in the G9G10G11G12 sequence. The $\mathrm{T}_{4}$ loop in acridine complexes shows much higher conformational variability than in uncomplexed structures. This variability is manifested by the presence of unusual conformations labeled $\mathrm{qA}, \mathrm{qB}, \mathrm{qC}$, and $\mathrm{qD}$ that are not homogenous enough to form distinct clusters but they do share several common structural characteristics. Conformation $\mathrm{qA}$ is typical by a glycosidic angle in the low anti $\left(\sim 200^{\circ}\right)$ region, $\beta+1$ torsion in $t\left(\sim 200^{\circ}\right)$ and $\alpha+1 / \gamma+1$ in $t / g+$ combination. Conformer qB is similar to the cluster 19 (A-DNA with $\alpha+1$ / $\gamma+1$ crank into the $t / t$ values, Table 1 ) but with a second sugar moiety in the canonical BI C2'-endo conformation. A common feature of the $\mathrm{qC}$ conformer is a presence of $\alpha+1 / \gamma+1$ torsions switched into the $g+/ g+$ values. $\mathrm{qD}$ conformation can be, based on $\delta$ and $\delta+1$ values, labeled as BI-like with $\alpha+1 / \gamma+1$ switched to the $g$-/ $t$ values, $\beta$ in $g+$, and with $\chi+1$ in the syn region.

Table 3 Characteristics of the six new conformational classes found by clustering

\begin{tabular}{|c|c|c|c|c|c|c|c|c|c|c|c|}
\hline Class ID & Description & $\mathbf{N}$ & $\delta$ & $\varepsilon$ & $\zeta$ & $a+1$ & $\beta+1$ & $\gamma+1$ & $\delta+1$ & $x$ & $x+1$ \\
\hline 35 & Bl-to- $A, \beta+1$ in $g+, a+1 / \gamma+1$ crank (high $t / t$ ), anti/low anti & 14 & 136 & 199 & 288 & 253 & 73 & 168 & 87 & 264 & 187 \\
\hline 97 & Bll-DNA, $a+1 / \gamma+1 \mathrm{crank}(\mathrm{t} / \mathrm{g}+)$, anti/low anti & 13 & 142 & 294 & 110 & 149 & 198 & 55 & 151 & 260 & 185 \\
\hline 113 & BI-DNA, $\varepsilon / \zeta$ in t/g+, $a+1 / \gamma+1$ crank $(g+/ t)$, anti/syn & 13 & 143 & 206 & 61 & 82 & 204 & 192 & 146 & 242 & 68 \\
\hline 114 & BI-DNA, $a+1 / \gamma+1$ crank (g-/g-), high $\beta+1$, anti/syn & 18 & 141 & 201 & 282 & 307 & 258 & 304 & 151 & 236 & 65 \\
\hline 115 & BI DNA, high $\varepsilon$, anti/low anti & 22 & 140 & 275 & 280 & 300 & 189 & 61 & 148 & 265 & 208 \\
\hline 117 & BI-DNA, $\beta+1$ in $g+, a+1 / \gamma+1$ crank (high $t / t$ ), anti/low anti & 19 & 139 & 196 & 286 & 249 & 73 & 172 & 145 & 263 & 211 \\
\hline
\end{tabular}

"Class ID" is a symbolic label of the class. "Description" is a short annotation of the class. " $\mathrm{N}$ " is the number of suites (dinucleotides) with the given class membership. Values of torsions represent the arithmetic means for the individual classes. Torsions are defined in Figure 2. 


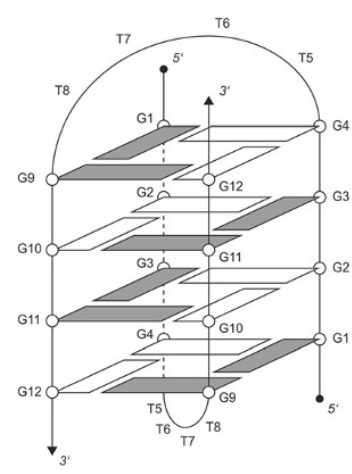

(a)

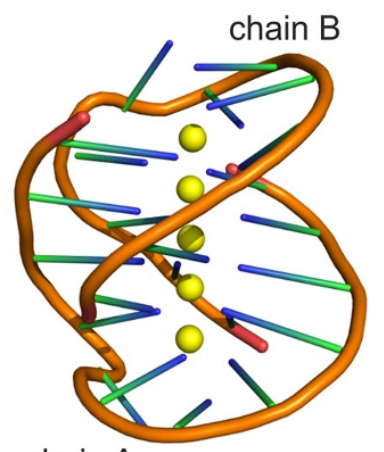

chain $\mathrm{A}$

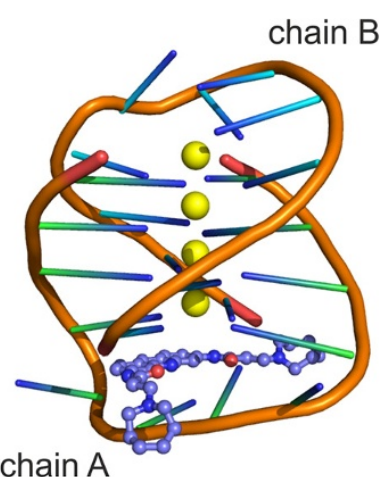

(c)

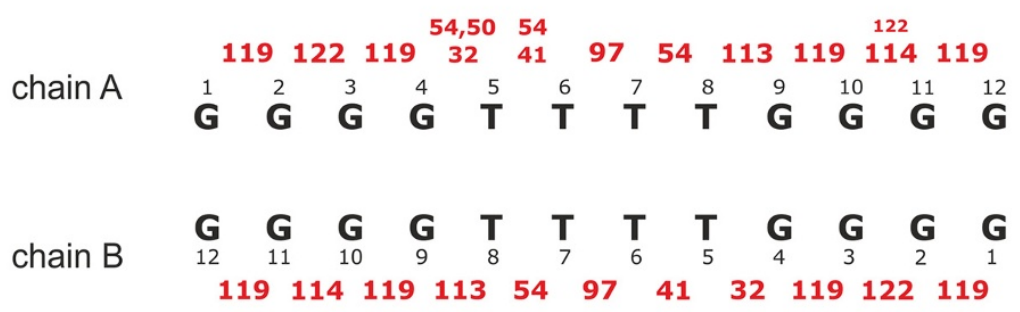

(d)

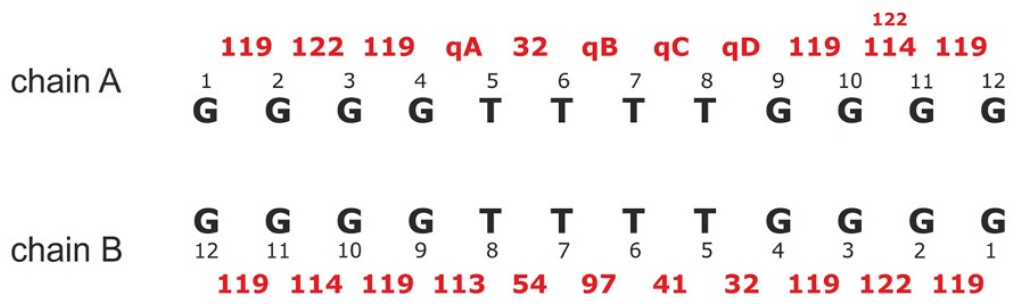

(e)

Figure 3 Oxytricha nova guanine quadruplex. (a) A schematic diagram of a double-stranded (bimolecular) guanine quadruplex from Oxytricha nova telomeric sequence $\left(\mathrm{G}_{4} \mathrm{~T}_{4} \mathrm{G}_{4}\right)_{2}$. A solid line represents a sugar-phosphate backbone. O. nova G-quadruplex has four $\mathrm{G}$-quartets formed from nucleotides in which syn and anti conformations of the glycosidic angle alternate along each strand [105]. Shaded rectangles indicate guanine residues in syn conformation (typically $X \sim 60^{\circ}-70^{\circ}$ ), clear rectangles indicate guanine residues in anti conformation (typically $X \sim 250^{\circ}-260^{\circ}$ ). $(\mathbf{b}) \mathrm{A}$ crystal structure of a bimolecular O. nova G-quadruplex 1.JPQ [104]. Overall topology is indicated by the orange ribbon. Bases are represented by green sticks, potassium ions stabilizing the whole structure are shown as yellow spheres. (c) A crystal structure of a complex of $O$. nova Gquadruplex with a drug acridine 3EUM [106]. Acridine affecting the conformation of a $\mathrm{T}_{4}$ loop in chain $\mathrm{A}$ is shown in blue. (d) Consensus conformational map of the O. nova G-quadruplex. By convention, chains are numbered in the 5'-to-3' direction. Conformational classes of individual dinucleotide steps are indicated by red numbers, their size is proportional to the frequency of their occurrence in investigated structures. A description of individual conformations is given in Tables 1 and 3. The T5T6 step adopts either a canonical BI conformation 54 if the G4T5 step is also in a canonical BI conformation, or an A-to-B conformation 41 if the G4T5 step is in a conformation 32. (e) Consensus conformational map of the $\mathrm{O}$. nova G-quadruplex complexed with a drug acridine. Individual conformations shown as red numbers are characterized in Tables 1 and 3.

Described conformational assignment demonstrates that O. nova G-quadruplexes are conformationally homogenous structures that could be decomposed into the clustered conformers some of which are unique to these structures (conformations 97, 113, 114, and 119). The complexation with the acridine molecule results in a higher conformational variability of the $\mathrm{T}_{4}$ loop compared to the $\mathrm{G}$-tracts.

\section{Conformation 115}

This class describes a conformation found exclusively in Holliday (four-way) junctions. It was noticed previously [32] as potentially existing, but only the larger data set including the recent data lead to its identification. It can be characterized as a BI-like conformer with unusually high $\varepsilon$ $\left(\sim 275^{\circ}\right)$ and A-like $X+1 \quad\left(\sim 208^{\circ}\right)$. This conformation is 
found in the sharp bend of the DNA strand between residues number 6 and number 7 (Figure 4).

\section{Conformation 117}

This class represents a BI-like conformation with both $\delta$ and $\delta+1$ torsions in the $\mathrm{C}^{\prime}$-endo region but its torsions $\alpha+1, \beta+1$ and $\gamma+1$ acquire values $\left(250^{\circ}, 73^{\circ}\right.$, and $172^{\circ}$, respectively) not typical for the BI conformer 54 . In addition, glycosidic torsion $x+1$ of the second residue is in A-like low anti region near $210^{\circ}$. This conformation was almost exclusively observed in protein/DNA complexes, about a half of them in complexes of nucleosome-core particle. The DNA bending induced by interactions between DNA and histone octamer has been explained [32] by the periodic alteration of BI and BII conformers with occasional insertion of conformation 116 (Table 1). The new conformation 117 is its rarer kin found only in some nucleosome structures located outside the protein/DNA interface.

\section{Conformation 35}

Class 35 can be characterized as a transitional BI-to-A conformation with the first residue in $\mathrm{BI}$ and the second residue resembling an A-form whose character is disturbed by unusual values of $\beta+1\left(g+, \sim 70^{\circ}\right), \alpha+1\left(\sim 250^{\circ}\right)$, and $\gamma+1$ torsions $\left(t, \sim 168^{\circ}\right)$. This conformation occurs in diverse protein/DNA complexes, about a half in DNA complexed with polymerases. Dinucleotides in this conformation are in direct contact with protein atoms via the phosphate charged oxygen.

\section{NMR structures}

We clustered a set of 12,300 dinucleotides from 664 NMR structures released before 15 February 2013 (see

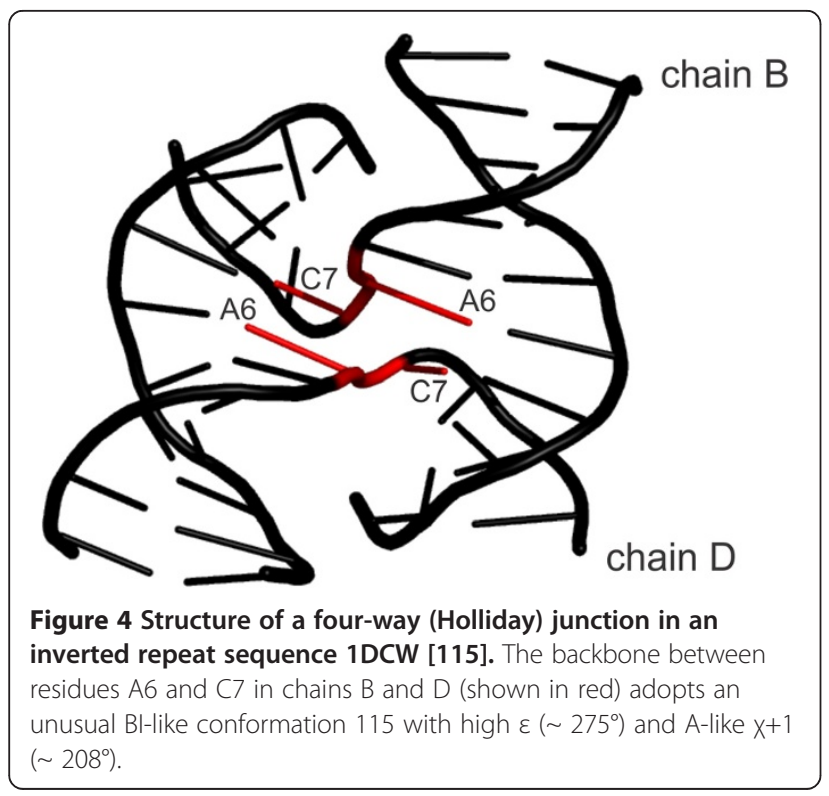

Additional file 1) utilizing $k$-NN procedure with $k=11$ and $\mathrm{v}_{\text {crit }}=0.001$. We assigned 11,313 dinucleotides (92\%), and subsequently applied a new round of clustering to the remaining 987 points. However, clustering did not reveal any new conformation that would be present in NMR and not in X-ray data.

Across-the-database assignment of dinucleotide conformers for $816 \mathrm{X}$-ray and 664 NMR DNA structures exhibit similar general features (Figure 5). The BI conformer 54 is dominant in both data sets, and the BI conformer 50, the BII conformers 86 and 96 , and several A-DNA conformers $(8,19,32,41)$ are also significantly populated. Similar qualitative features of the assignment of the local DNA backbone conformers demonstrate that DNA in solution and in the crystal phase, which is highly hydrated, show similar behavior. However similar the overall features are, both populations also exhibit significant differences. Perhaps the most noticeable is the difference of the overall BI population (the conformers $50+54+116$ ) that forms $65 \%$ in NMR, and only $47 \%$ in crystal structures. The BI conformers are more populated in NMR than in crystal structures, striking is especially a large population of the conformer 50 in NMR (27\%, compared to just $11 \%$ in crystals). Also the fractions of some other conformers differ significantly. NMR structures have more populated the mixed B-A conformer 32 , and crystal structures the canonical Aform 8 , the mixed A-B form 41, and the BII conformers 96 and 86. NMR structures have a slightly larger proportion of unassigned dinucleotides than crystal structures, $8 \%$ versus $5.4 \%$.

Reason for the above-mentioned differences between NMR and crystal structures is not obvious, and we propose just a few possible explanations. Protein/DNA complexes form $65 \%$ of structures resolved by X-ray crystallography, but this fraction is only $17 \%$ in NMR. The higher number of protein/DNA complexes resolved by X-ray crystallography could perhaps explain a larger number of the A-form in crystal than in NMR structures as the A-form is often induced by interactions with proteins. A larger population of BI and a smaller population of BII in NMR structures cannot be explained so easily. Either of these forms has only limited sequence preferences, and there seem to be no obvious rationale supporting a hypothesis that crystal packing favors the BII over the BI conformation. A different hypothetical explanation could lie in the process of interpretation of the NMR experimental data. Their relative scarcity caused by the low density of protons, and sometimes equivocal interpretation of experiments such as indirect spin-spin couplings ("J-couplings") may cause uncertainties especially in the assignment of torsions $\alpha$ and $\zeta$ of the phosphodiester linkage [116]. The resulting DNA structure may then be influenced by the refinement protocol in which 


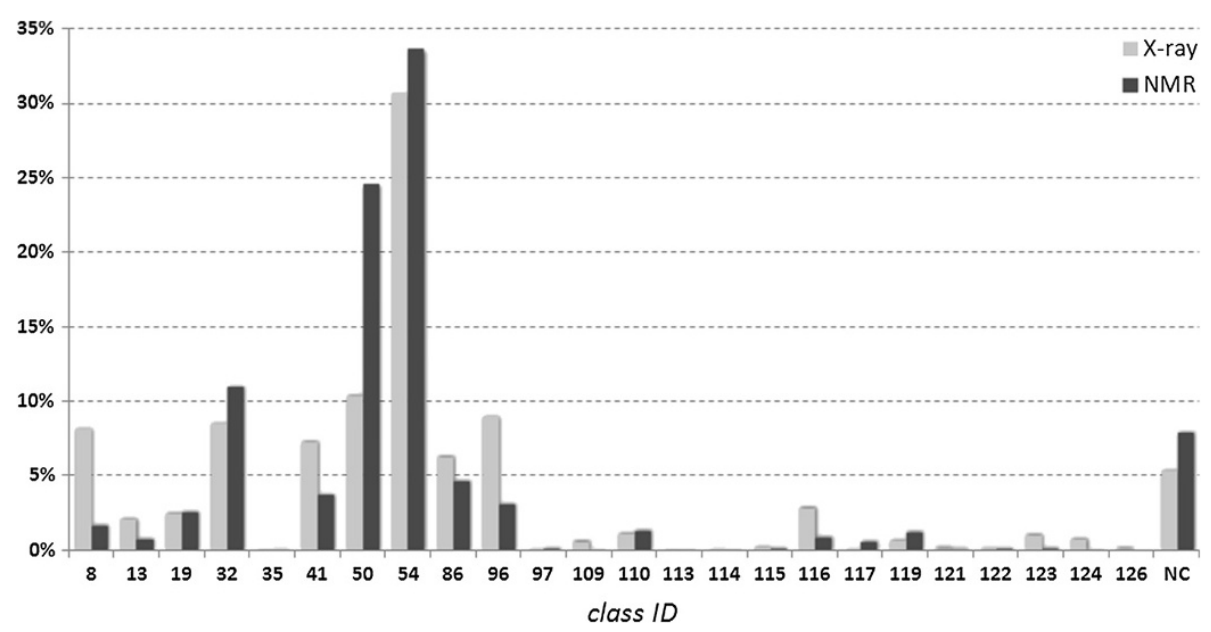

Figure 5 Comparison of a fraction of individual conformational classes (Tables 1 and 3) identified in structures resolved by X-ray ( 816 structures) and NMR techniques (664 structures).

the experimental restrains are combined with force fields in a computer simulation. Relatively low number of the experimental restraints and imperfection of the force fields, namely their incorrectly set torsion preferences, may perhaps favor BI over BII forms.

\section{Conclusions}

In the present work we investigated several supervised machine-learning approaches (ridge regression (RR), multi-layer perceptron (MLP) neural network, radial basis function (RBF) neural network, and $k$ nearest neighbors $(k-\mathrm{NN}))$ to develop a protocol for an automatic classification of local DNA conformations. The classifiers were trained and tested using the previously published manually classified set of dinucleotides [32]. Various parameters of the machine learning methods were set to their optimum values utilizing a 10-fold cross-validation procedure. According to the results of our testing, the best method is $k$-nearest neighbor. This technique not only achieves high classification accuracy, but also allows identifying conformers that cannot be assigned to any of known classes. We subsequently investigated the unassigned conformers for the presence of new clusters using a modified clustering method based on the leader algorithm [89]. By the proposed machine learning workflow (Figure 1) we successfully analyzed Xray and NMR structures of both naked and complexed DNA released until 15 February 2013. In addition to 18 conformational classes compiled in [32] we identified 6 new classes in X-ray structures, and no additional new classes in NMR data. We assigned four of these conformers to two structurally important DNA families: guanine quadruplexes and Holliday (four-way) junctions. The new clusters enhance structural annotation of $O$. nova telomeric G-quadruplex [32] and we were able to construct its consensus conformational map (Figure 3(d) and (e)). Comparison of frequencies of individual conformers found in X-ray and NMR structures showed that they have similar qualitative features so that DNA in the crystal phase and in solution populate the same regions of the conformational space. Observed differences between populations of X-ray and NMR conformers can be partially assigned to different composition of both datasets, partially to the refinement protocol of NMR structures that may favor BI over the BII form.

\section{Additional files}

Additional file 1: Data sets. DatasetF contains 4,567 dinucleotide "suite" units classified previously [32] into 18 classes. We used this classification as a "gold standard" in the present work. DatasetF was stratifically divided into the training set (sheet "DatasetF_train") and into the test set (sheet "DatasetF_test"). Sheet "X-ray data" contains all crystallography data, and sheet "NMR data" contains all NMR data analyzed in the current work.

Additional file 2: Confusion matrices. Confusion matrices of the ridge regression (RR), the multi-layer perceptron (MLP) neural network, the radial basis function (RBF) neural network and the $k$ nearest neighbors ( $k$-NN). The "true" class [32] is shown in the rows, and the class predicted by the given method is shown in the columns.

\section{Competing interests}

The authors declare that they have no competing interests.

\section{Authors' contributions}

PČ designed and implemented MLP and RBF neural network models, performed tests of all used methods and drafted the manuscript. DS instigated the study, participated in its coordination, implemented the k-NN method, annotated new conformers, and drafted the manuscript. BS was responsible for data selection and data processing, performed the analyses of NMR data, and drafted the manuscript. JK implemented and applied the regularised regression method. JC participated in data selection, data processing, and implemented additional support scripts. All authors read and approved the final manuscript. 


\section{Acknowledgements}

This project has been supported by the Research grants MSM 6046137306, MSM 6046137302 and financial support from specific university research (MSMT No 21/2011). BS and JC are supported by the Czech Science Foundation, grant P305/12/1801, and by the institutional grant AV0Z50520701.

\section{Author details}

'Laboratory of Informatics and Chemistry, ICT Prague, Technická 5, Prague 6 166 28, Czech republic. ²Department of Computing and Control Engineering, ICT Prague, Technická 5, Prague 6166 28, Czech republic. ${ }^{3}$ Faculty of Nuclear Sciences and Physical Engineering, CTU Prague, Trojanova 13, Prague 2122 00, Czech republic. ${ }^{4}$ Institute of Biotechnology AS CR, v. v. i., Vídeňská 1083, Prague 4142 00, Czech republic.

Received: 28 November 2012 Accepted: 28 May 2013 Published: 25 June 2013

\section{References}

1. Watson JD, Crick FHC: Molecular structure of nucleic acids - a structure for deoxyribose nucleic acid. Nature 1953, 171(4356):737-738.

2. Drew HR, Wing RM, Takano T, Broka C, Tanaka S, Itakura K, Dickerson RE: Structure of a B-DNA dodecamer: conformation and dynamics. Proc Natl Acad Sci USA 1981, 78(4):2179-2183.

3. Wang $A H$, Fujii $\mathrm{S}$, van Boom JH, Rich A: Molecular structure of the octamer d(G-G-C-C-G-G-C-C): modified A-DNA. Proc Natl Acad Sci USA 1982, 79(13):3968-3972

4. McCall M, Brown T, Kennard O: The crystal structure of d(G-G-G-G-C-C-CC). A model for poly(dG).poly(dC). J Mol Biol 1985, 183(3):385-396.

5. Wang AHJ, Quigley GJ, Kolpak FJ, Crawford JL, Vanboom JH, Vandermarel G, Rich A: Molecular-structure of a left-handed double helical DNA fragment at atomic resolution. Nature 1979, 282(5740):680-686.

6. Drew HR, Dickerson RE: Structure of a B-DNA dodecamer. III. Geometry of hydration. J Mol Biol 1981, 151(3):535-556.

7. Calladine CR: Mechanics of sequence-dependent stacking of bases in B-DNA. J Mol Biol 1982, 161(2):343-352.

8. Jones $S$, van Heyningen $P$, Berman HM, Thornton JM: Protein-DNA interactions: a structural analysis. J Mol Biol 1999, 287(5):877-896.

9. Lu XJ, Shakked Z, Olson WK: A-form conformational motifs in ligandbound DNA structures. J Mol Biol 2000, 300(4):819-840.

10. Lejeune D, Delsaux N, Charloteaux B, Thomas A, Brasseur R: Protein-nucleic acid recognition: statistical analysis of atomic interactions and influence of DNA structure. Proteins 2005, 61(2):258-271.

11. Nekludova L, Pabo CO: Distinctive DNA conformation with enlarged major groove is found in Zn-finger-DNA and other protein-DNA complexes. Proc Natl Acad Sci USA 1994, 91(15):6948-6952.

12. Olson WK, Gorin AA, Lu XJ, Hock LM, Zhurkin VB: DNA sequencedependent deformability deduced from protein-DNA crystal complexes. Proc Natl Acad Sci USA 1998, 95(19):11163-11168.

13. Tolstorukov MY, Jernigan RL, Zhurkin VB: Protein-DNA hydrophobic recognition in the minor groove is facilitated by sugar switching. $J \mathrm{Mol}$ Biol 2004, 337(1):65-76.

14. Murphy FV, Churchill ME: Nonsequence-specific DNA recognition: a structural perspective. Structure 2000, 8(4):R83-R89.

15. Shakked Z, Guzikevich-Guerstein G, Frolow F, Rabinovich D, Joachimiak A, Sigler PB: Determinants of repressor/operator recognition from the structure of the trp operator binding site. Nature 1994, 368(6470):469-473.

16. Kim Y, Geiger JH, Hahn S, Sigler PB: Crystal structure of a yeast TBP/TATAbox complex. Nature 1993, 365(6446):512-520.

17. Guzikevich-Guerstein G, Shakked Z: A novel form of the DNA double helix imposed on the TATA-box by the TATA-binding protein. Nat Struct Biol 1996, 3(1):32-37

18. Lebrun A, Shakked Z, Lavery R: Local DNA stretching mimics the distortion caused by the TATA box-binding protein. Proc Natl Acad Sci USA 1997, 94(7):2993-2998.

19. Ding J, Das K, Hsiou Y, Sarafianos SG, Clark AD Jr, Jacobo-Molina A, Tantillo C, Hughes SH, Arnold E: Structure and functional implications of the polymerase active site region in a complex of HIV-1 RT with a doublestranded DNA template-primer and an antibody Fab fragment at $2.8 \mathrm{~A}$ resolution. J Mol Biol 1998, 284(4):1095-1111.
20. Pelletier H, Sawaya MR, Kumar A, Wilson SH, Kraut J: Structures of ternary complexes of rat DNA polymerase beta, a DNA template-primer, and ddCTP. Science 1994, 264(5167):1891-1903.

21. Eom SH, Wang J, Steitz TA: Structure of Taq polymerase with DNA at the polymerase active site. Nature 1996, 382(6588):278-281.

22. Kiefer JR, Mao C, Braman JC, Beese LS: Visualizing DNA replication in a catalytically active Bacillus DNA polymerase crystal. Nature 1998, 391(6664):304-307.

23. Doublie S, Tabor S, Long AM, Richardson CC, Ellenberger T: Crysta structure of a bacteriophage T7 DNA replication complex at $2.2 \mathrm{~A}$ resolution. Nature 1998, 391(6664):251-258.

24. Pavletich NP, Pabo CO: Crystal structure of a five-finger GLI-DNA complex: new perspectives on zinc fingers. Science 1993, 261(5129):1701-1707.

25. Robinson H, Gao YG, McCrary BS, Edmondson SP, Shriver JW, Wang AH: The hyperthermophile chromosomal protein Sac7d sharply kinks DNA. Nature 1998, 392(6672):202-205

26. Winkler FK, Banner DW, Oefner C, Tsernoglou D, Brown RS, Heathman SP, Bryan RK, Martin PD, Petratos K, Wilson KS: The crystal structure of EcoRV endonuclease and of its complexes with cognate and non-cognate DNA fragments. EMBO J 1993, 12(5):1781-1795.

27. Horton NC, Perona JJ: Recognition of flanking DNA sequences by EcoRV endonuclease involves alternative patterns of water-mediated contacts. J Biol Chem 1998, 273(34):21721-21729.

28. Kostrewa D, Winkler FK: Mg2+ binding to the active site of EcoRV endonuclease: a crystallographic study of complexes with substrate and product DNA at 2 A resolution. Biochemistry-Us 1995, 34(2):683-696.

29. Travers AA: Reading the minor groove. Nat Struct Biol 1995, 2(8):615-618.

30. Choo Y, Klug A: Physical basis of a protein-DNA recognition code. Curr Opin Struct Biol 1997, 7(1):117-125.

31. Elrod-Erickson M, Benson TE, Pabo CO: High-resolution structures of variant Zif268-DNA complexes: implications for understanding zinc finger-DNA recognition. Structure 1998, 6(4):451-464.

32. Svozil D, Kalina J, Omelka M, Schneider B: DNA conformations and their sequence preferences. Nucleic Acids Res 2008, 36(11):3690-3706.

33. Moravek Z, Neidle S, Schneider B: Protein and drug interactions in the minor groove of DNA. Nucleic Acids Res 2002, 30(5):1182-1191.

34. Oguey C, Foloppe N, Hartmann B: Understanding the sequencedependence of DNA groove dimensions: implications for DNA interactions. PLoS One 2010, 5(12):e15931.

35. Orbons LP, van der Marel GA, van Boom JH, Altona C: Hairpin and duplex formation of the DNA octamer d(m5C-G-m5C-G-T-G-m5C-G) in solution. An NMR study. Nucleic Acids Res 1986, 14(10):4187-4196.

36. Jain A, Wang G, Vasquez KM: DNA triple helices: biological consequences and therapeutic potential. Biochimie 2008, 90(8):1117-1130.

37. Stuhmeier F, Welch JB, Murchie Al, Lilley DM, Clegg RM: Global structure of three-way DNA junctions with and without additional unpaired bases: a fluorescence resonance energy transfer analysis. Biochemistry-Us 1997, 36(44):13530-13538.

38. Hays FA, Watson J, Ho PS: Caution! DNA crossing: crystal structures of Holliday junctions. J Biol Chem 2003, 278(50):49663-49666.

39. Burge S, Parkinson GN, Hazel P, Todd AK, Neidle S: Quadruplex DNA: sequence, topology and structure. Nucleic Acids Res 2006, 34(19):5402-5415.

40. Rippe K, Jovin TM: Parallel-stranded duplex DNA. Methods Enzymol 1992, 211:199-220.

41. Bhattacharyya $D$, Bansal M: Local variability and base sequence effects in DNA crystal structures. J Biomol Struct Dyn 1990, 8(3):539-572.

42. Gorin AA, Zhurkin VB, Olson WK: B-DNA twisting correlates with base-pair morphology. J Mol Biol 1995, 247(1):34-48.

43. Hunter CA, Lu XJ: DNA base-stacking interactions: a comparison of theoretical calculations with oligonucleotide X-ray crystal structures. J Mol Biol 1997, 265(5):603-619.

44. Strahs D, Schlick T: A-Tract bending: insights into experimental structures by computational models. J Mol Biol 2000, 301(3):643-663.

45. Tolstorukov MY, Colasanti AV, McCandlish DM, Olson WK, Zhurkin VB: A novel roll-and-slide mechanism of DNA folding in chromatin: implications for nucleosome positioning. J Mol Biol 2007, 371(3):725-738.

46. Battistini F, Hunter CA, Gardiner EJ, Packer MJ: Structural mechanics of DNA wrapping in the nucleosome. J Mol Biol 2010, 396(2):264-279.

47. Olson WK, Bansal M, Burley SK, Dickerson RE, Gerstein M, Harvey SC, Heinemann U, Lu XJ, Neidle S, Shakked Z, et al: A standard reference frame for the description of nucleic acid base-pair geometry. J Mol Biol 2001, 313(1):229-237. 
48. Arnott $S$, Selsing E: Structures for the polynucleotide complexes poly $(\mathrm{dA})$ with poly (dT) and poly(dT) with poly(dA) with poly (dT). J Mol Biol 1974, 88(2):509-521

49. Vlieghe D, Van Meervelt L, Dautant A, Gallois B, Precigoux G, Kennard O: Parallel and antiparallel (G.GC)2 triple helix fragments in a crystal structure. Science 1996, 273(5282):1702-1705.

50. Rhee S, Han Z, Liu K, Miles HT, Davies DR: Structure of a triple helical DNA with a triplex-duplex junction. Biochemistry-Us 1999, 38(51):16810-16815.

51. Neidle S, Balasubramanian S: Quadruplex Nucleic Acids. Cambridge: RSC Publishing; 2006

52. von Kitzing E, Lilley DM, Diekmann S: The stereochemistry of a four-way DNA junction: a theoretical study. Nucleic Acids Res 1990, 18(9):2671-2683.

53. Lilley DM: Structures of helical junctions in nucleic acids. Q Rev Biophys 2000, 33(2):109-159.

54. Reshetnikov RV, Kopylov AM, Golovin AV: Classification of g-quadruplex DNA on the basis of the quadruplex twist angle and planarity of $\mathrm{g}$ quartets. Acta Naturae 2010, 2(4):72-81.

55. Watson J, Hays FA, Ho PS: Definitions and analysis of DNA Holliday junction geometry. Nucleic Acids Res 2004, 32(10):3017-3027.

56. Neidle S: Principles of Nucleic Acid Structure. Oxford: Academic; 2007.

57. Dickerson RE: Base sequence and helix structure variation in $B$ and $A$ DNA. J Mol Biol 1983, 166(3):419-441.

58. Yanagi K, Prive GG, Dickerson RE: Analysis of local helix geometry in three B-DNA decamers and eight dodecamers. J Mol Biol 1991, 217(1):201-214.

59. Suzuki M, Amano N, Kakinuma J, Tateno M: Use of a 3D structure data base for understanding sequence-dependent conformational aspects of DNA. J Mol Biol 1997, 274(3):421-435.

60. ElHassan MA, Calladine CR: Conformational characteristics of DNA: Empirical classifications and a hypothesis for the conformational behaviour of dinucleotide steps. Philos T Roy Soc A 1997, 355(1722):43-100.

61. Packer MJ, Hunter CA: Sequence-dependent DNA structure: the role of the sugar-phosphate backbone. J Mol Biol 1998, 280(3):407-420.

62. Schuerman GS, Van Meervelt L: Conformational flexibility of the DNA backbone. J Am Chem Soc 2000, 122(2):232-240.

63. Varnai $P$, Djuranovic D, Lavery R, Hartmann B: Alpha/gamma transitions in the B-DNA backbone. Nucleic Acids Res 2002, 30(24):5398-5406.

64. Djuranovic D, Hartmann B: Conformational characteristics and correlations in crystal structures of nucleic acid oligonucleotides: evidence for substates. J Biomol Struct Dyn 2003, 20(6):771-788.

65. Djuranovic D, Hartmann B: DNA fine structure and dynamics in crystals and in solution: the impact of $\mathrm{BI} / \mathrm{BI}$ l backbone conformations. Biopolymers 2004, 73(3):356-368.

66. Djuranovic D, Oguey C, Hartmann B: The role of DNA structure and dynamics in the recognition of bovine papillomavirus E2 protein target sequences. J Mol Biol 2004, 339(4):785-796.

67. Madhumalar A, Bansal M: Sequence preference for BI/BII conformations in DNA: MD and crystal structure data analysis. J Biomol Struct Dyn 2005, 23(1):13-27.

68. Heddi B, Foloppe N, Bouchemal N, Hantz E, Hartmann B: Quantification of DNA BI/BII backbone states in solution. Implications for DNA overall structure and recognition. J Am Chem Soc 2006, 128(28):9170-9177.

69. Marathe A, Karandur D, Bansal M: Small local variations in B-form DNA lead to a large variety of global geometries which can accommodate most DNA-binding protein motifs. BMC Struct Biol 2009, 9:24.

70. Schneider B, Neidle S, Berman HM: Conformations of the sugar-phosphate backbone in helical DNA crystal structures. Biopolymers 1997, 42(1):113-124.

71. Sims GE, Kim SH: Global mapping of nucleic acid conformational space: dinucleoside monophosphate conformations and transition pathways among conformational classes. Nucleic Acids Res 2003, 31(19):5607-5616.

72. Elsawy KM, Hodgson MK, Caves LS: The physical determinants of the DNA conformational landscape: an analysis of the potential energy surface of single-strand dinucleotides in the conformational space of duplex DNA. Nucleic Acids Res 2005, 33(18):5749-5762.

73. Sargsyan K, Wright J, Lim C: GeoPCA: a new tool for multivariate analysis of dihedral angles based on principal component geodesics. Nucleic Acids Res 2012, 40(3):e25.

74. Wijmenga SS, van Buuren BNM: The use of NMR methods for conformational studies of nucleic acids. Prog Nucl Magn Reson Spectrosc 1998, 32(4):287-387.

75. Furtig B, Richter C, Wohnert J, Schwalbe H: NMR spectroscopy of RNA. Chembiochem Eur J Chem Biol 2003, 4(10):936-962.
76. Zidek L, Stefl R, Sklenar V: NMR methodology for the study of nucleic acids. Curr Opin Struct Biol 2001, 11(3):275-281.

77. Gorenstein DG, Schroeder SA, Fu JM, Metz JT, Roongta V, Jones CR: Assignments of 31P NMR resonances in oligodeoxyribonucleotides: origin of sequence-specific variations in the deoxyribose phosphate backbone conformation and the 31P chemical shifts of double-helical nucleic acids. Biochemistry-Us 1988, 27(19):7223-7237.

78. Schroeder SA, Roongta V, Fu JM, Jones CR, Gorenstein DG: Sequencedependent variations in the 31P NMR spectra and backbone torsional angles of wild-type and mutant Lac operator fragments. Biochemistry-Us 1989, 28(21):8292-8303.

79. el antri S, Bittoun P, Mauffret O, Monnot M, Convert O, Lescot E, Fermandjian S: Effect of distortions in the phosphate backbone conformation of six related octanucleotide duplexes on CD and 31P NMR spectra. Biochemistry-Us 1993, 32(28):7079-7088.

80. Heddi B, Foloppe N, Oguey C, Hartmann B: Importance of accurate DNA structures in solution: the Jun-Fos model. J Mol Biol 2008, 382(4):956-970

81. Abi-Ghanem J, Heddi B, Foloppe N, Hartmann B: DNA structures from phosphate chemical shifts. Nucleic Acids Res 2010, 38(3):e18.

82. Nikolova EN, Bascom GD, Andricioaei I, Al-Hashimi HM: Probing sequencespecific DNA flexibility in a-tracts and pyrimidine-purine steps by nuclear magnetic resonance (13)C relaxation and molecular dynamics simulations. Biochemistry-Us 2012, 51(43):8654-8664.

83. Chou SH, Cheng JW, Reid BR: Solution structure of [d(ATGAGCGAATA)]2. Adjacent G:A mismatches stabilized by cross-strand base-stacking and Bll phosphate groups. J Mol Biol 1992, 228(1):138-155.

84. Lefebvre A, Mauffret $O$, Lescot E, Hartmann B, Fermandjian S: Solution structure of the $\mathrm{CpG}$ containing $\mathrm{d}(\mathrm{CTTCGAAG}) 2$ oligonucleotide: NMR data and energy calculations are compatible with a BI/BIl equilibrium at CpG. Biochemistry-Us 1996, 35(38):12560-12569.

85. Tisne C, Hantz E, Hartmann B, Delepierre M: Solution structure of a nonpalindromic 16 base-pair DNA related to the HIV-1 kappa B site: evidence for $\mathrm{BI}-\mathrm{Bll}$ equilibrium inducing a global dynamic curvature of the duplex. J Mol Biol 1998, 279(1):127-142.

86. Wecker K, Bonnet MC, Meurs EF, Delepierre M: The role of the phosphorus BI-BII transition in protein-DNA recognition: the NF-kappaB complex. Nucleic Acids Res 2002, 30(20):4452-4459.

87. Heddi B, Oguey C, Lavelle C, Foloppe N, Hartmann B: Intrinsic flexibility of BDNA: the experimental TRX scale. Nucleic Acids Res 2010, 38(3):1034-1047.

88. Schneider B, Moravek Z, Berman HM: RNA conformational classes. Nucleic Acids Res 2004, 32(5):1666-1677

89. Hartigan JA: Clustering Algorithms. John Wiley \& Sons Inc; 1975.

90. Berman HM, Olson WK, Beveridge DL, Westbrook J, Gelbin A, Demeny T, Hsieh SH, Srinivasan AR, Schneider B: The nucleic acid database. A comprehensive relational database of three-dimensional structures of nucleic acids. Biophys J 1992, 63(3):751-759.

91. Murray LJ, Arendall WB 3rd, Richardson DC, Richardson JS: RNA backbone is rotameric. Proc Natl Acad Sci USA 2003, 100(24):13904-13909.

92. Mu Y, Nguyen PH, Stock G: Energy landscape of a small peptide revealed by dihedral angle principal component analysis. Proteins 2005, 58(1):45-52.

93. Altis A, Nguyen PH, Hegger R, Stock G: Dihedral angle principal component analysis of molecular dynamics simulations. J Chem Phys 2007, 126(24):244111.

94. Jammalamadaka SR, Sengupta A: Topics in Circular Statistics. Singapore: World Scientific Pub Co Inc; 2001

95. Hoerl AE WKR: Ridge regression: biased estimation for nonorthogonal problems. Technometrics 1970, 42(1):7.

96. Bishop CA: Pattern Recognition and Machine Learning. 2nd edition. New York: Springer; 2006

97. Cohen J: A coefficient of agreement for nominal scales. Educ Psychol Meas 1960, 20(1):10

98. MacCuish DJ, MacCuish EN: Clustering in Bioinformatics and Drug Discovery 3rd edition. Boca Raton: CRC Press; 2010.

99. Neidle $S$ : The structures of quadruplex nucleic acids and their drug complexes. Curr Opin Struct Biol 2009, 19(3):239-250.

100. Williamson JR: G-quartet structures in telomeric DNA. Annu Rev Biophys Biomol Struct 1994, 23:703-730.

101. Sen D, Gilbert W: Formation of parallel four-stranded complexes by guanine-rich motifs in DNA and its implications for meiosis. Nature 1988, 334(6180):364-366. 
102. Huppert JL, Balasubramanian S: G-quadruplexes in promoters throughout the human genome. Nucleic Acids Res 2007, 35(2):406-413.

103. Horvath MP, Schultz SC: DNA G-quartets in a 1.86 A resolution structure of an Oxytricha nova telomeric protein-DNA complex. J Mol Biol 2001, 310(2):367-377.

104. Haider S, Parkinson GN, Neidle S: Crystal structure of the potassium form of an Oxytricha nova G-quadruplex. J Mol Biol 2002, 320(2):189-200.

105. Smith FW, Feigon J: Quadruplex structure of Oxytricha telomeric DNA oligonucleotides. Nature 1992, 356(6365):164-168.

106. Campbell NH, Patel M, Tofa AB, Ghosh R, Parkinson GN, Neidle S: Selectivity in ligand recognition of G-quadruplex loops. Biochemistry-Us 2009, 48(8):1675-1680

107. Haider SM, Parkinson GN, Neidle S: Structure of a G-quadruplex-ligand complex. J Mol Biol 2003, 326(1):117-125.

108. Theobald DL, Schultz SC: Nucleotide shuffling and ssDNA recognition in Oxytricha nova telomere end-binding protein complexes. EMBO J 2003, 22(16):4314-4324.

109. Gill ML, Strobel SA, Loria JP: Crystallization and characterization of the thallium form of the Oxytricha nova G-quadruplex. Nucleic Acids Res 2006, 34(16):4506-4514

110. Campbell NH, Smith DL, Reszka AP, Neidle S, O'Hagan D: Fluorine in medicinal chemistry: beta-fluorination of peripheral pyrrolidines attached to acridine ligands affects their interactions with $\mathrm{G}$-quadruplex DNA. Org Biomol Chem 2011, 9(5):1328-1331.

111. Schultze P, Smith FW, Feigon J: Refined solution structure of the dimeric quadruplex formed from the Oxytricha telomeric oligonucleotide $d$ (GGGGTTTGGGG). Structure 1994, 2(3):221-233.

112. Smith FW, Schultze $P$, Feigon J: Solution structures of unimolecular quadruplexes formed by oligonucleotides containing Oxytricha telomere repeats. Structure 1995, 3(10):997-1008.

113. Schultze P, Hud NV, Smith FW, Feigon J: The effect of sodium, potassium and ammonium ions on the conformation of the dimeric quadruplex formed by the Oxytricha nova telomere repeat oligonucleotide $\mathrm{d}(\mathrm{G}(4) \mathrm{T}$ (4)G(4)). Nucleic Acids Res 1999, 27(15):3018-3028.

114. Gill ML, Strobel SA, Loria JP: 205TI NMR methods for the characterization of monovalent cation binding to nucleic acids. J Am Chem Soc 2005, 127(47):16723-16732.

115. Eichman BF, Vargason JM, Mooers BH, Ho PS: The Holliday junction in an inverted repeat DNA sequence: sequence effects on the structure of four-way junctions. Proc Natl Acad Sci USA 2000, 97(8):3971-3976.

116. Sychrovsky V, Vokacova Z, Sponer J, Spackova N, Schneider B: Calculation of structural behavior of indirect NMR spin-spin couplings in the backbone of nucleic acids. J Phys Chem B 2006, 110(45):22894-22902.

doi:10.1186/1471-2105-14-205

Cite this article as: Čech et al:: Automatic workflow for the classification of local DNA conformations. BMC Bioinformatics 2013 14:205.

\section{Submit your next manuscript to BioMed Central and take full advantage of:}

- Convenient online submission

- Thorough peer review

- No space constraints or color figure charges

- Immediate publication on acceptance

- Inclusion in PubMed, CAS, Scopus and Google Scholar

- Research which is freely available for redistribution

Submit your manuscript at www.biomedcentral.com/submit
Ciomed Central 